\title{
Let's Use Haptics!
}

\author{
http://dx.doi.org/10.3991/ijoe.v9iS8.3392 \\ M. R. Quintas, M. T. Restivo, J. Rodrigues and P. Ubaldo \\ University of Porto, Porto, Portugal
}

\begin{abstract}
The concept and the use of haptic devices need to be disseminated and they should become familiar among young people. At present haptics are used in many everyday tasks in different fields.
\end{abstract}

Additionally, their use in interaction with virtual reality applications simulating real systems sense of touch will increase the users' realism and immersion and, consequently, they will contribute to improve the intrinsic knowledge to the simulations' goals.

However, haptics are associated with expensive equipment and usually they offer several degrees of freedom. The objective of this work is to make their cost not much more expensive than a "special" mouse by offering a low cost solution with just one degree of freedom (1DOF) useful in many simple cases.

Additionally, it is also an objective of this work the development of simple virtual reality systems requiring interactions only requiring one degree of freedom.

A low cost, single-axis force-feedback haptic device of 1 degree of freedom has been developed. For evaluating the interest of this prototype a "Spring Constant" application was built and used as a demonstrator. The complete system - the haptic interacting with the "Spring Constant" - will be described in the present work.

Index Terms-Haptic interfaces; Sensors; User interfaces; Virtual reality.

\section{INTRODUCTION}

The interfaces between a human operator and a machine are the human senses. Sight and hearing have been explored for decades based in video and audio. More recently the sense of touch became more explored in the technological area by using the so called haptic devices. By the provision of means for vision, hearing and touch the operator can achieve higher degree of immersion and even has the chance to combine them with $3 \mathrm{D}$ virtual reality in order to develop virtual systems with a closer approach to the real environment.

Haptics are also essential in tele-presence where man controls robotic systems in task performance using vision, sound and force feedback as it was done in 1996 with the historic Sojourner rover on Mars [1], even taking into account problems related with information transmission delay. In medicine the applications are many and in industry too, as is the case of automotive and aerospace industries, design, optimization and assembly processes, etc.

As haptics are modern devices integrated in emergent technologies used in many areas it is relevant to introduce the concept to the younger at an early stage. However, those devices present high costs while also offering a degree of sophistication that largely exceeds the needs for simple educational applications. Their performance capabilities are, therefore, related with high cost. Among their characteristics the number of degrees-of-freedom (DOF) is, often, higher than what is required for simple applications.

A low cost, single-axis force-feedback joystick (1DOF) was developed to be used at undergraduate level. Ref. [2] reports the use of haptic devices to interact with virtual applications based on dynamic systems allowing students to understand and to analyse different dynamic system concepts like damping, stiffness, and inertia. Ref. [3] addresses the initiative and interest in engaging youngsters at K-12 level and the public in general in the use of haptics and the familiarization with this concept. The present work is motivated to pursue similar strategies with the following additional goals, namely:

- To develop 1DOF haptic device built with reused and recycled old parts from obsolete computer and printer equipment;

- To get a hardware solution at a very low cost not adding more than $20 €$ to that of the recycled parts;

- To incentive a do-it-yourself attitude in all those excited with the idea.

The next section will describe the system.

\section{A Simple 1 DOF Haptic Device}

\section{A. Working principle}

Usually, haptics are developed to interact within working environments where velocities are very low. They are normally built based in the use of servo-motors. Therefore the motors are typically working near the stall torque zone.

In the present work the goal is to employ obsolete equipment and particularly over dimensioned DC motors which will be used to work in the stall torque zone. These motors are able to produce a torque proportional to the applied voltage.

Being the control system based in a microcontroller (PIC18F2431 from Microchip) an encoder is used to measure the displacement imposed by the user. This value will give to the microcontroller the information of the force whenever the displacement is associated to physical model behavior.

Since the torque is proportional to the applied voltage, the microcontroller will know the voltage value which must be applied to the motor. A pulse with modulation method (PWM) is used for driving an $\mathrm{H}$-bridge motor drive (L298N).

A quadrature encoder interface module (QEI) is incorporated in the PIC18F2431 microcontroller and this is crucial for reading the encoder value, Fig. 1 . The selection 
of this component has been due to the fact that it is the only PIC ${ }^{\circledR}$ in the 8 bit family providing this module.

The proposed working principle for this 1DOF haptic device is to work with the electric motor in the stall torque zone (null angular velocity). And so enough torque has to be applied to force the motor to stop rotating. At this stage it will draw the maximum amount of current - stalling current. Overheating is then a serious risk since the rotor is immobilized and so there is not any intrinsic ventilation. However, the use of over dimension electric motors solves the problem.

For the interaction with virtual reality a second microcontroller (PIC18F2550) is needed to interface the communication (SPI and USB 2.0) between both systems.

\section{B. Main features}

In the present prototype the translation of the linear motion carriage is made by a pulley and a timing belt transmission chain is driven by a permanent magnetic DC motor shaft. The total path is of $70 \mathrm{~mm}$ - Error! Reference source not found. The system is able to deliver over $20 \mathrm{~N}$, [4].

For testing the efficiency and for evaluating the acceptance by the target publics a simple virtual model named "Spring Constant" was conceived and developed. This application is described in the next paragraph.

\section{1 DOF haptic interaction with virtual environment}

When the user imposes a displacement to the haptic the microcontroller will communicate it to the virtual environment in a PC software application embedded in the respective physical model.

Then the application will feedback to the haptic the force information and the device may feedback it to the user. At the same time the user will observe through the graphical interface screen the model response to the displacement imposed by him/herself.

For the spring model three zones were defined: the "free zone", the "working zone" and the "wall zone".

In the "free zone" the displacement does not cause any contact with the spring. The "working zone", the spring compression zone, is where the haptic displacement will compress the virtual spring. When the spring is totally compressed the "wall zone" is reached. The "wall effect" has been created by imposing to the user the maximum force delivered by the motor, Fig. 3 .

\section{SOFTWARE DEMONSTRATOR}

In the present work it will be briefly described the "Spring Constant" application mainly devoted to STEM students. It explores the Spring Constant concept of different materials (spring steel, aluminum and brass) and its relation with mechanical and geometric parameters (wire diameter, spring diameter and transverse elastic modulus or shear modulus). The user interface of the "Spring Constant" system presents three sections.

The one on the right side shows a cantilever spring in compression mode. The spring exhibits different aspects in accordance with the selected parameters. It will show the spring deformation under haptic actuation.

On the left side there are two sections. In the upper one, the user may select the spring wire diameter and spring diameter. This selection together with the spring material

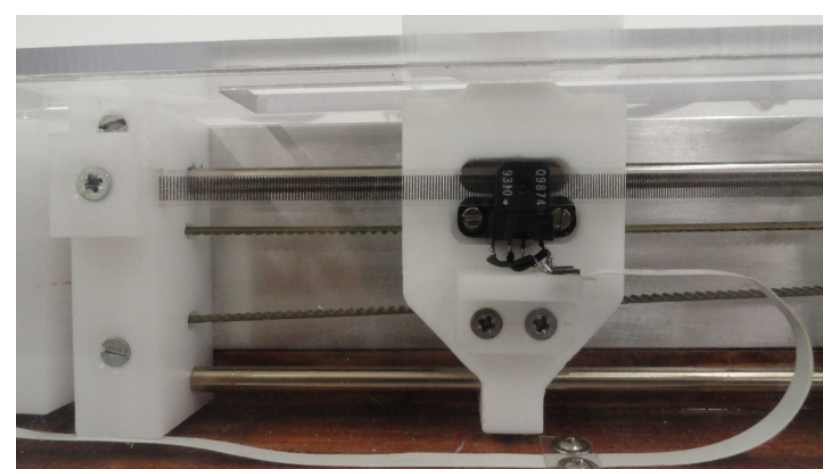

Figure 1. Encoder for displacement measurement.



Figure 2. 1 DOF haptic device.

will define the spring constant. Geometric details are also shown in this section permitting to relate the spring constant to the referred parameters. In the lower left section the graphic displays the $\mathbf{F}(\Delta \mathrm{x})$ diagram whose slope is the spring constant for each of the selected springs. It also exhibits the previous $\mathbf{F}(\Delta \mathrm{x})$ diagram making possible to compare the difference caused by the new selected parameters. The user interface is shown in Figs. 3a), b), c) and d).

On the top of the interface a "start process" button allows the user to start the interaction with the virtual cantilevered spring by using the haptic device. Once the haptic actuates the free end of the spring, this will be under compression and the force will increase proportionally to the displacement of the spring free end. A red dot will appear along the straight line of the $\mathbf{F}(\Delta \mathrm{x})$ diagram according to the values of the applied force, $\mathbf{F}$, and the spring free end displacement, $\Delta \mathrm{x}$.

The next set of figures shows the system - the $1 \mathrm{DOF}$ haptic device and the "Spring Constant" user interface -, Fig. 3a), the user interface of a specific selected steel spring not under compression, Fig. 3b), the user interface of that selected spring fully compressed, Fig. 3c) and, finally, the user interface of a specific selected brass spring by choosing selectable spring parameters and materials in order to obtain a lower elastic constant, Fig.3d).

In the graphical window representing the function $\mathrm{F}(\Delta \mathrm{x})$, the red dot follows the haptic movement, i.e., the displacement imposed at the free end of the spring by the haptic device, by "compressing it". The gray line represents the elastic constant of the spring previously selected. 


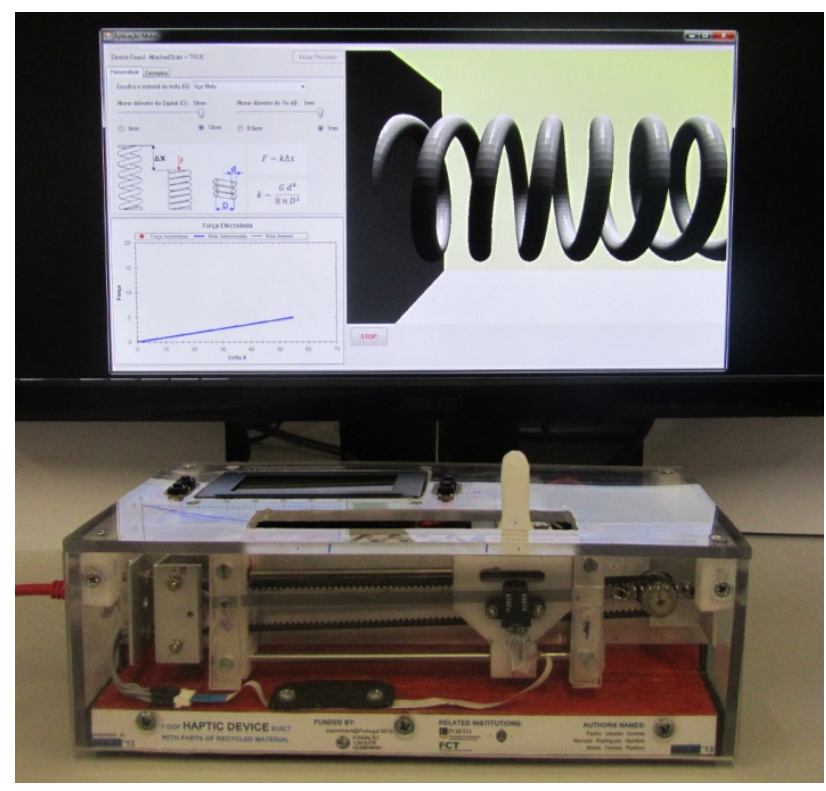

Figure 3. a) Haptic device and the "Spring Constant" application.

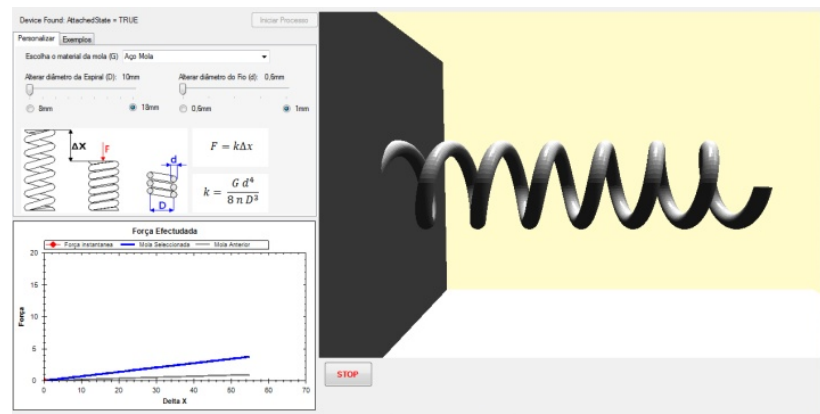

Figure 3. b) "Spring Constant" user interface (not actuated steel spring).

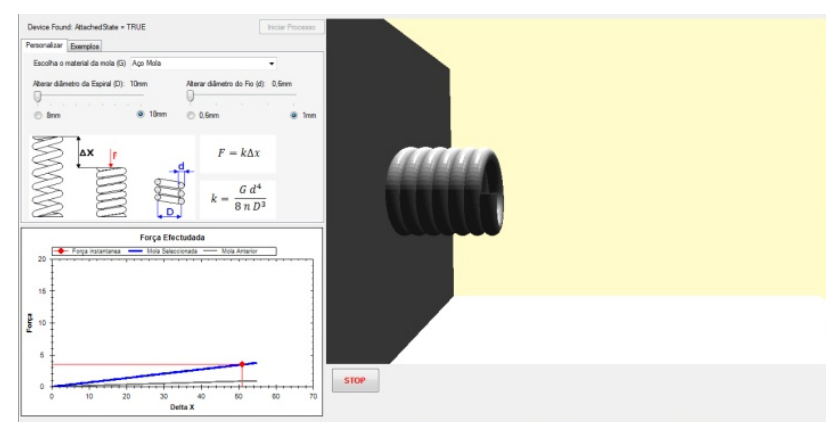

Figure 3. c) "Spring Constant" user interface (steel spring fully compressed)

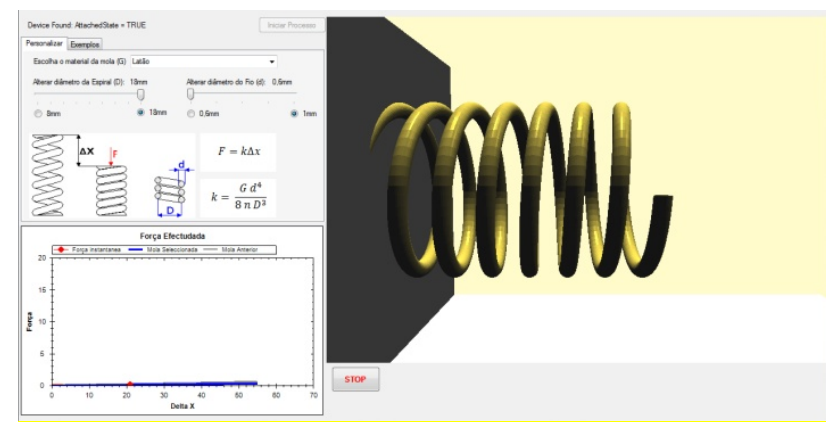

Figure 3. d) "Spring Constant" user interface for a brass spring.

\section{FINAL COMMENTS}

The developed low cost, single-axis force-feedback haptic device of One Degree of Freedom (1 DOF) has proved its working performance interacting with a virtual reality system and presenting a maximum force of around $20 \mathrm{~N}$. This is, a range similar to that of many of the commercial haptic devices.

The system has been demonstrated in different contexts, not only at STEM level but also in the university environment and in the Exhibition Session of Experiment@International Conference, Coimbra, Portugal, 2013.

Considering the very low cost of this device making it easily affordable, it is possible to make the application online available for distance interaction users or to be downloaded for institutional or personal user computers. Therefore, the haptic device may also be used to remote actuation of any experimental set-up in which one direction actuation concept is adaptable.

It has been received with a very positive reaction by the different target audiences whenever they experienced that they were "felling the spring force". Additional evaluations will be carried out in lecturing environments during the present academic year.

During all demonstration sessions it was recognized the users' interest and enthusiastic attitude and it has been confirmed their unfamiliarity with the concept of haptic systems, contributing in this way for its dissemination.

\section{ACKNOWLEDGMENT}

The present work is due to the development and innovation activities in online experimentation within the project Experiment@Portugal 2102 funded by the Calouste Gulbenkian Foundation and in agreement with other activities related with the use of emergent technologies within PEst-OE/EME/LA0022/2013, FCT.

\section{REFERENCES}

[1] A. Fisch, C. Mavroidis, J. Melli-Huber and Y. Bar-Cohen, "Biologically-Inspired Intelligent Robots, Chapter 4: Haptic Devices for Virtual Reality", Telepresence, and Human-Assistive Robotics. SPIE Press, 2003.

[2] D.I. Grow, L.N. Verner and A.M. Okamura, "Educational Haptics", accessed in May 2013, http://www.aaai.org/Papers/ Symposia/Spring/2007/SS-07-09/SS07-09-012.pdf

[3] C.E. Wong and A.M. Okamura, "The Snaptic Paddle: A Modular Haptic Device http://dx.doi.org/10.1109/WHC.2005.135

[4] P. U. Gomes, M. R. Quintas and M. T. Restivo, "1 DOF haptic device built with parts of recycled material", Proceedings of the Experiment@International Conference, Coimbra 18-20, Portugal, 2013.

\section{AUTHORS}

M. R. Quintas and M. T. Restivo are with the IDMEC-FEUP research center and with Faculty of Engineering of University of Porto, Porto, Portugal (mrq@fe.up.pt, trestivo@fe.up.pt).

J. Rodrigues and P. Ubaldo, are with Faculty of Engineering of University of Porto, Porto, Portugal (jose.c.rodrigues@gmail.com).

This article is an extended and modified version of a paper presented at the International Conference exp.at'13, held 18-20 September 2013, in Coimbra, Portugal. Submitted 18 November 2013. Published as resubmitted by the authors 04 December 2013. 